\title{
Canonical and Non-canonical Genomic Imprinting in Rodents
}

\author{
Hisato Kobayashi* \\ Department of Embryology, Nara Medical University, Kashihara, Japan
}

\section{OPEN ACCESS}

Edited by:

Anthony Isles,

Cardiff University, United Kingdom

Reviewed by:

Ferdinand Von Meyenn,

ETH Zürich, Switzerland

Courtney W. Hanna,

University of Cambridge,

United Kingdom

Philippe Arnaud,

GReD, CNRS-Université Clermont Auvergne-INSERM, France

*Correspondence:

Hisato Kobayashi

hiskobay@naramed-u.ac.jp

Specialty section:

This article was submitted to

Developmental Epigenetics,

a section of the journal

Frontiers in Cell and Developmental

Biology

Received: 24 May 2021

Accepted: 16 July 2021

Published: 05 August 2021

Citation:

Kobayashi H (2021) Canonical and Non-canonical Genomic

Imprinting in Rodents.

Front. Cell Dev. Biol. 9:713878.

doi: 10.3389/fcell.2021.713878
Genomic imprinting is an epigenetic phenomenon that results in unequal expression of homologous maternal and paternal alleles. This process is initiated in the germline, and the parental epigenetic memories can be maintained following fertilization and induce further allele-specific transcription and chromatin modifications of single or multiple neighboring genes, known as imprinted genes. To date, more than 260 imprinted genes have been identified in the mouse genome, most of which are controlled by imprinted germline differentially methylated regions (gDMRs) that exhibit parent-of-origin specific DNA methylation, which is considered primary imprint. Recent studies provide evidence that a subset of gDMR-less, placenta-specific imprinted genes is controlled by maternalderived histone modifications. To further understand DNA methylation-dependent (canonical) and -independent (non-canonical) imprints, this review summarizes the loci under the control of each type of imprinting in the mouse and compares them with the respective homologs in other rodents. Understanding epigenetic systems that differ among loci or species may provide new models for exploring genetic regulation and evolutionary divergence.

Keywords: genomic imprinting, DNA methylation, non-canonical imprinting, histone modification, rodent, germline differentially methylated region, mouse genome, epigenetics

\section{RODENTS: SYMBOLIC MODELS IN BIOMEDICAL AND GENETIC RESEARCH}

Rodents such as mice and rats are commonly used as representative laboratory animals. The genomes of these organisms have been progressed along with the human genome project; thus, the C57BL/6 mouse (Mus musculus) and Brown Norway rat (Rattus norvegicus) become the second and third mammals to have their genomes sequenced in 2002 and 2004, respectively (Waterston et al., 2002; Gibbs et al., 2004). Their genomes of approximately three billion base pairs each contains roughly the same number of genes as the human genome. Furthermore, almost all human genes associated with diseases have counterparts in the rodent genome, which appear highly conserved throughout mammalian evolution. Thus, these experimental rodents generally deepen our understanding of mammalian genetic and (epi-)genomic regulatory systems.

Mammals are diploid organisms arising from the fusion of two parental gametes, with each donating one set of autosomal chromosomes (19 autosomes in mice, 20 in rats, and 22 in humans) plus one set of sex chromosome (X or Y) to the offspring. According to Mendel's law, diploid

Abbreviations: DNMT, DNA methyltransferase; gDMR, germline differentially methylated region; ICR, imprinting control region; lncRNA, long non-coding RNA; LTR, long terminal repeat; piRNA, Piwi-interacting RNA; sDMR, secondary differentially methylated region. 
cells contain parental copies of each autosomal gene, which are predicted to show the same transcription state. However, "genomic imprinting" is a form of non-Mendelian inheritance that results in parent-of-origin allele-specific gene expression of autosomal loci or of loci on the diploid $\mathrm{X}$ chromosome (only in extra-embryonic tissues in females). Polymorphism information between strains or individuals helps distinguish between paternal and maternal alleles. It was only natural that the phenomenon of genomic imprinting was discovered and well-investigated in mice, where nuclear transfer and genetic engineering technologies have always been developed and where numerous strains (polymorphisms between strains can serve as genetic markers of parent-of-origin in allele-specific analysis) have been established and maintained.

\section{DISCOVERY OF GENOMIC IMPRINTING IN MAMMALS}

In 1984, two laboratories published landmark papers that reported a new phenomenon in mammalian genome biology (Barton et al., 1984; McGrath and Solter, 1984). Both studies independently performed pronuclear transfer experiments from fertilized mouse eggs to produce androgenetic and gynogenetic embryos containing only sperm-derived or oocyte-derived chromosome sets. These "uniparental" embryos could not survive to term but could develop to some extent with sex-specific developmental abnormalities. Androgenetic embryos preferentially develop extra-embryonic and placental structures at the expense of embryo development. Conversely, gynogenetic embryos (or parthenogenetic embryos by artificial activation of oocytes) have poor growth of placental lineages and developmental arrest, possibly due to extra-embryonic defects. These opposite phenotypes underlie the functional differences in developmental genes in paternal and maternal genomes. The mice that were bred to have uniparental disomies, in which either single or partial chromosomes are inherited from only one parent, for individual chromosomes also show aberrant phenotypes, such as overgrowth, growth retardation, or abnormal behavior (Cattanach, 1986).

Nevertheless, not all chromosomes produce abnormalities when present as disomies, depending on which chromosome or part is made uniparental; however, those commonly lead to striking phenotypic differences. These investigations revealed the requirement of both maternal and paternal genomes for normal development, which was tied to an intriguing biological phenomenon called genomic imprinting. Uniparental inheritance of the genome or chromosome occurs spontaneously in humans, resulting in early pregnancy losses, like androgenetic and parthenogenetic conceptuses (hydatidiform moles and benign ovarian teratomas), or moderate to severe developmental disabilities, known as imprinting diseases (Linder et al., 1975; Kajii and Ohama, 1977; Wake et al., 1978; Kalish et al., 2014). Subsequent evolutionary and genetic studies of imprinted loci have shown that this phenomenon is present only in placental mammals among vertebrates.
The surprising finding of these studies was that mammalian genes could function differentially depending on whether they originated from the mother or father. Before the study of uniparental disomies, a "maternal-effect" locus called Tme (Tassociated maternal effect) was identified on the proximal mouse chromosome 17 overlapping deletions of maternal-effect lethal mutants, like $T^{h p}$ or $T^{\text {lub2 }}$ (Johnson, 1974; Winking and Silver, 1984). The region was later revealed to be the locus of $\operatorname{Ig} f 2 r$, expressed exclusively from the maternally inherited allele; therefore, its expression is dependent on the "parent-of-origin." Simultaneously, the closely linked $\mathrm{H} 19$ and Igf2 genes, which are reciprocally imprinted, were identified in mouse chromosome 7; H19 produces a long non-coding RNA (lncRNA) exclusively expressed from the maternal allele, and Igf2 originates from the opposite allele. Interestingly, the opposite imprinting of Igf2 and its scavenging receptor gene, Igf2r, demonstrates conflicting parental effects of growth promotion and growth restriction, which supports the classic "parent-offspring conflict theory" for the evolution of genomic imprinting (Trivers, 1974; Moore and Haig, 1991).

\section{CANONICAL GENOMIC IMPRINTING IS MEDIATED BY MATERNAL OR PATERNAL DNA METHYLATION}

The discovery of the first endogenous imprinted genes in 1991 (Barlow et al., 1991; Bartolomei et al., 1991; DeChiara et al., 1991), which were differentially expressed from the maternal and paternal alleles, sparked initial efforts to elucidate the mechanisms of imprint establishment, maintenance, and erasure that together control the timing and placement of genomic imprinting. One prominent candidate of the nonMendelian system is epigenetic regulation, in which DNA methylation (mainly occurs in $\mathrm{CpG}$ dinucleotides) is the most studied mechanism and has been shown to play a key role in mouse models of genomic imprinting and fetal reprogramming. A strong link between DNA methylation and imprinting regulation has been indicated by the cases of imprinted transgenic mouse lines. In a few of these mice the foreign transgene becomes methylated in a parent-specific manner in the gamete, inherited with parent-of-origin specific methylation into the diploid cells of embryo, and subsequently, the modification is erased and reestablished upon passage through the germ line (Chaillet et al., 1991).

Allele-specific DNA methylation of imprinted regions, also known as imprinted germline differentially methylated regions (gDMRs), has been studied as the best candidate for the molecular mechanism of inheriting parental-specific imprints following fertilization. Because parental imprints must be established when the parental genomes can be distinguished, investigators assayed methylation acquisition during gametogenesis, when the maternal and paternal genomes are entirely separated and can be independently epigenetically modified. Paternalspecific methylation of the gDMRs at three imprinted loci (H19 and subsequently discovered Dlk1-Meg3 and Rasgrf1) is acquired prenatally in prospermatogonia before the onset of 
meiosis in the male germline (Davis et al., 2000; Kato et al., 2007). In contrast, maternal-specific gDMR methylation occurs postnatally in growing oocytes, with different gDMRs (at least 21 maternal gDMRs have been identified in mice) that are methylated at a slightly different time during oocyte growth (Lucifero et al., 2004; Hiura et al., 2006). In both germlines, DNA methylation is established through the action of de novo DNA methyltransferase (DNMT) 3a and the accessory protein DNMT3L (Bourc'his et al., 2001; Hata et al., 2002; Kaneda et al., 2004). Although it is unclear how specific sequences are chosen for allele-specific DNA methylation in the germline, recent studies have demonstrated that histone modification across gDMR sequences provides an essential instructive step for DNMT proteins (Figures 1A,B). In oogenesis, the transcriptiondependent histone marker H3K36me3 (trimethylation of histone $\mathrm{H} 3$ at lysine 36) guides DNA methylation over active gene bodies, leading to the establishment of all maternal methylation imprints (Kobayashi et al., 2012a; Veselovska et al., 2015; Xu et al., 2019). Transcription start sites in oocytes are often oocytespecific (carried in part by retroviral promoters) and upstream of canonical promoters and imprinted DMRs, hence transcriptioncoupled DNA methylation spans these domains in an oocytespecific manner (Chotalia et al., 2009; Brind'Amour et al., 2018). In fetal spermatogenesis, H3K36me2 (dimethylation of H3K36) shapes the gene body and intergenic DNA methylation and guides paternal methylation at the gDMRs (Shirane et al., 2020). Only in Rasgrf1 gDMR, the Piwi-interacting RNA (piRNA) pathway and the rodent-specific DNMT3C are also responsible for the establishment of paternal DNA methylation (Watanabe et al., 2011; Barau et al., 2016). In addition to imprinted gDMRs, there are more than a thousand promoters or CpG islands on non-imprinted genes that are differentially methylated between oocytes and sperm; however, the vast majority lose their differential marks during epigenetic reprogramming events during early embryogenesis (Smallwood et al., 2011; Kobayashi et al., 2012a). After fertilization, the paternal genome is actively demethylated before the first DNA replication, whereas the maternal genome is passively demethylated throughout several rounds of DNA replication until the blastocyst stage. Imprinted gDMRs are protected from these erasure events by recruiting maintenance DNMT1 and accessory UHRF1 through the recognition of a methylated sequence motif by the zincfinger proteins, ZFP57 and ZNF445, along with the interaction of TRIM28 with histone methyltransferases (Sharif et al., 2007; Hirasawa et al., 2008; Quenneville et al., 2011; Messerschmidt et al., 2012; Takahashi et al., 2019).

At the end of 2018, at least 260 coding and non-coding genes were found to be imprinted, and 24 imprinted gDMRs were identified in the mouse genome (Tucci et al., 2019). Many of these gDMRs act as imprinting control regions (ICRs) regulating the monoallelic expression of the neighboring solo imprinted gene and clusters of imprinted genes. The majority of maternal ICRs directly regulate a promoter for either a messenger RNA or a lncRNA by silencing one allele by DNA methylation. In contrast, paternal ICRs are not located at promoters but rather map to intergenic regions. However, the imprinting of gene clusters often involves locus-specific and complex molecular mechanisms, such as transcriptional silencing by an antisense transcript and allele-specific chromatin changes at target genes or cis-regulatory elements by the ICRs (Hark et al., 2000; Terranova et al., 2008; Latos et al., 2012). These imprinted genes under the control of ICRs also act as barriers to prevent mammalian embryos from parthenogenesis (Kono et al., 2004; Kawahara et al., 2007; Li et al., 2018). Thus, parent-of-origin specific DNA methylation, also called "canonical imprinting," is considered a primary imprint marker that directly or indirectly controls most imprinted genes, which are responsible for the abnormalities of uniparental disomies or embryos.

\section{NON-CANONICAL IMPRINTING IS MEDIATED BY MATERNAL HISTONE MODIFICATION}

Although DNA methylation has been known to specify imprinting, the possibility that histone modifications in the gametes could also determine imprinting has also been demonstrated (Okae et al., 2014). A subset of imprinted genes is specifically paternally expressed in the placenta but not imprinted in the embryo, and the establishment of a part of such imprinted genes is independent of oocyte-specific DNA methylation, as DNMT-deficiency in growing oocytes did not affect the imprinted paternal expression of these genes in the extra-embryonic lineage (Chen et al., 2019; Hanna et al., 2019). The key gametic imprinting mark of the "non-canonical" (DNA methylation-independent) imprinting is the repressive histone mark $\mathrm{H} 3 \mathrm{~K} 27 \mathrm{me} 3$ (trimethylation of $\mathrm{H} 3$ at lysine 27) in the oocyte, which was found to transiently imprint several loci within pre-implantation (Inoue et al., 2017a). Furthermore, H2AK119ub1 (mono-ubiquitinated histone H2A at lysine 119) was highly colocalized with $\mathrm{H} 3 \mathrm{~K} 27 \mathrm{me} 3$ in oocytes, which is equalized mainly at the two-cell stage but guides maternal H3K27me3 inheritance after fertilization (Chen et al., 2021; Mei et al., 2021). Thus, H2AK119ub1 and H3K27me3, which are catalyzed by the polycomb repressive complexes (PRC1 and PRC2), mediate maternal allele-specific silencing of at least seven imprinted genes, namely Sfmbt2, Phf17, Gab1, Sall1, Platr20 (5133400J02Rik), Smoc1, and Slc38a4, in mice (Figure 1C), several of which have been previously shown to play important roles in placental function and development (Itoh et al., 2000; Miri et al., 2013; Matoba et al., 2019). Maternal H3K27me3 and H2AK119ub1 are not maintained beyond pre-implantation development (Hanna et al., 2019; Chen et al., 2021; Mei et al., 2021), and transition to a more permanent epigenetic state is required to preserve paternal expression during post-implantation development (Inoue et al., 2017a; Chen et al., 2019; Hanna et al., 2019). The long terminal repeats (LTRs) of endogenous retroviral elements can act as alternative promoters for non-canonical imprinted genes and paternal allele-specific accumulation of the active histone mark $\mathrm{H} 3 \mathrm{~K} 4 \mathrm{me} 3$ (trimethylation of $\mathrm{H} 3$ at lysine 4) occurs at these LTR promoters (Hanna et al., 2019). Finally, these LTRs are methylated on the maternal allele in extra-embryonic tissues; thus, maternally inherited H3K27me3 
A Canonical paternal imprinting (e.g., H19) Fetal spermatogenesis

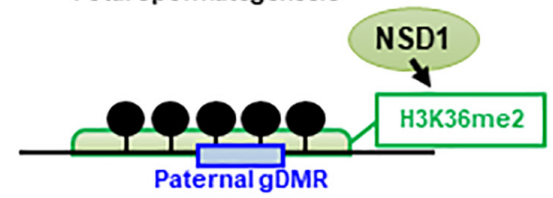

Embryonic and extraembryonic lineages

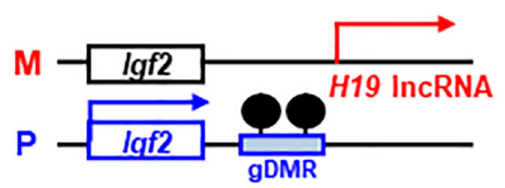

B Canonical maternal imprinting (e.g., Impact)

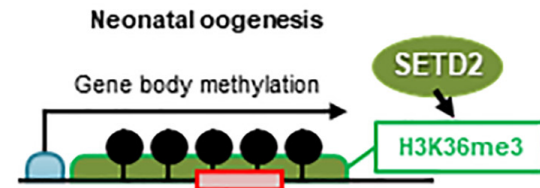

Matemal gDMR
Embryonic and extraembryonic lineages

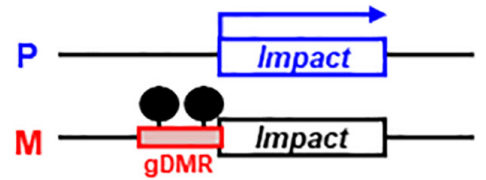

C Non-canonical imprinting (e.g., Gab1)
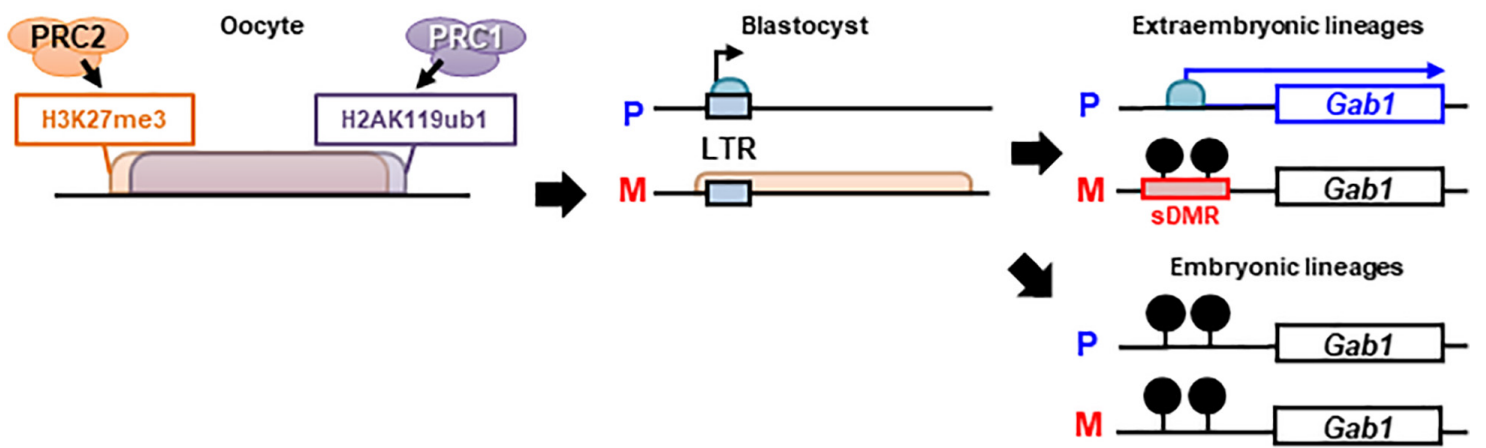

D Zdbf2, canonical but unique imprinting (transient gDMR and continuous sDMR)

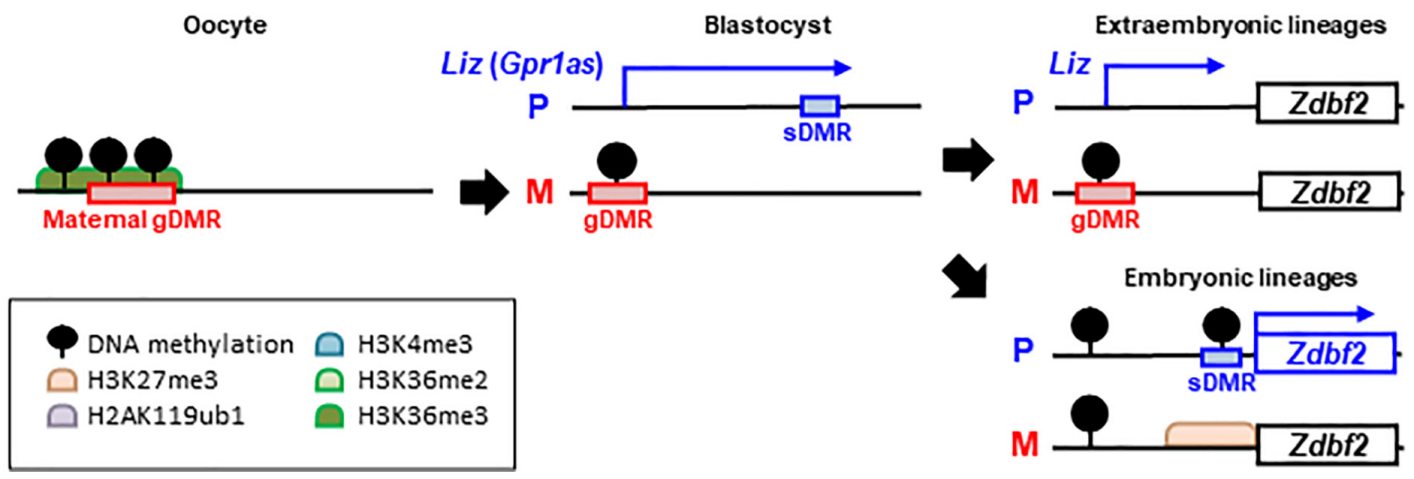

FIGURE 1 | Epigenetic mechanisms of canonical and non-canonical imprinted gene regulation. There are several examples of different epigenetic inheritance patterns between paternal $(\mathrm{P})$ and maternal $(\mathrm{M})$ alleles, that control paternally expressed protein-coding genes. (A) Paternal and (B) maternal germline differentially methylated region (gDMR)-mediated canonical imprinting. H3K36 methyltransferases NSD1 and SETD2 have been shown to establish H3K36me2 or H3K36me3 in pro-spermatogonia or oocytes and to be required for de novo DNA methylation at paternal or maternal gDMRs, respectively. In general, canonical imprinting is stably maintained throughout somatic life and mediates monoallelic silencing of imprinted genes or non-coding RNAs. (C) Non-canonical imprinting, such as Gab1 loci. H2AK119ub1 guides maternal inheritance and zygotic deposition of H3K27me3, and thus, maternally inherited H3K27me3 is maintained until the blastocyst (pre-implantation) stage. Then, maternal H3K27me3 silences the LTR retrotransposon-derived alternative promoter, which becomes actively transcribed on only the paternal allele. Although maternal $\mathrm{H} 3 \mathrm{~K} 27 \mathrm{me} 3$ is lost after implantation, maternal allele-specific DNA methylation is established as an imprinted sDMR in extra-embryonic tissues, and thus, monoallelic paternal expression of non-canonically imprinted LTRs and nearby protein-coding genes can be maintained. In the post-implantation epiblast, these ERVs are silenced by DNA methylation in both alleles, resulting in a loss-of-imprinting in somatic lineages (not shown in the figure). (D) Zdbf2 locus is a unique example of secondary imprinting. Transient paternal allele-specific expression of a long isoform transcript of Zdbf2 (Liz, also called Gpr1as) originated from the maternal gDMR, occurs in the pre-implantation embryo. Liz continues to be paternally expressed by the persistence of the maternal gDMR in extra-embryonic tissues. In the embryonic tissues, maternal gDMR is biallelically methylated and loses its imprinted status and Liz transcription; However, Zdbf2 retains imprinted expression because of acquired paternal DNA methylation at the sDMR and active H3K4me3 at the Zdbf2 promoter via traversing Liz transcription during gastrulation. 
imprinting transitions to imprinted DNA methylation at the secondary DMRs (sDMRs) and can act as a long-term imprinting in placental linage.

Notably, oocyte-derived H3K27me3 also serves as a maternal imprint for the lncRNA Xist, triggering paternal X chromosome inactivation in mouse female pre-implantation embryos and extra-embryonic tissues (Inoue et al., 2017b). Like noncanonical imprinting at autosomal loci, $\mathrm{X}$ inactivation can be clonally inherited and suppress the entire chromosome through several epigenetic suppression pathways (Chen and Zhang, 2020). In addition to H3K27me3 imprinting, failure of $\mathrm{X}$ chromosome inactivation results in embryonic lethality, emphasizing the developmental importance of these interrelated processes. However, the functional and molecular relationship between $\mathrm{H} 3 \mathrm{~K} 27 \mathrm{me} 3$-mediated non-canonical imprinting at autosomes and imprinted $\mathrm{X}$ chromosome inactivation or what distinguishes these strategies for biological diversity from DNA methylation-based canonical imprinting remains unresolved.

\section{SECONDARY DMRS: A LESSON FROM ZDBF2 IMPRINTED GENE}

Unlike gDMRs, imprinted sDMRs acquire allele-specific DNA methylation during embryonic development, rather than inheriting it from germ cells. Although secondary DMRs do not function as primary imprinting markers, allele-specific methylation of these regions frequently corresponds to gene silencing in a tissue-specific manner, such as Cdkn1c (Fan et al., 2005; Wood et al., 2010). Although sDMRs may play a role in maintaining imprinted expression (John and Lefebvre, 2011; Kobayashi et al., 2012b), they remain untested in most regions. The majority of sDMRs at canonical imprinted loci have been identified to be located within the imprinted genes or clusters and acquire allele-specific methylation by the hierarchical regulation of the gDMRs (Stoger et al., 1993; Lopes et al., 2003; Yamasaki et al., 2005; Williamson et al., 2011; Mohammad et al., 2012; Greenberg et al., 2017; Saito et al., 2018). One mechanism across several imprinted loci is the presence of a monoallelic transcript from gDMR passing through regulatory elements such as promoters and CpG islands (Ferguson-Smith, 2011). Consequently, DNMT3B targets sites of transcriptional elongation (Baubec et al., 2015), resulting in the acquisition of DNA methylation along the transcribed allele. As not all DMRs are located within transcribed regions, there must also be alternative mechanisms to establish allelic methylation at secondary loci.

Differences in the acquisition of sDMRs between embryonic and extra-embryonic lineages have been observed across several canonical imprinted domains (Lewis et al., 2004; Sato et al., 2011; Duffie et al., 2014). In particular, the DMR dynamics observed at $Z d b f 2$ highlight epigenetic changes in these developmental processes (Figure 1D). Zdbf2 is a canonical, but unique, imprinted gene with paternal expression and, paradoxically, a paternal DMR near its promoter [the paradoxical finding of the paternal DMR adjacent to a paternally expressed gene was later explained through serial experiments systematically ablating epigenetic modifiers (Greenberg et al., 2017)]. Early studies of Zdbf2 suggested that paternal DMR might be a gDMR because the DMR is methylated in the sperm and not in oocytes (Kobayashi et al., 2009). However, subsequent studies in embryos showed that paternal DNA methylation was erased in pre-implantation embryos and reset secondarily during post-implantation development (Kobayashi et al., 2012b; Duffie et al., 2014). This paternal sDMR was established by the transient monoallelic expression of a long isoform of Zbdf2 ( $\mathrm{Liz}$, also called GPR1AS in humans) originating from an upstream transcription start site, which is regulated by a maternal gDMR (Kobayashi et al., 2012b, 2013; Greenberg et al., 2017). Thus, Liz-induced sDMR can be maintained in embryonic lineage and lead to postnatal paternal expression of $Z d b f 2$. Meanwhile, Liz transcription is lost with the subsequent monoallelic to biallelic DNA methylation switch of the upstream maternal gDMR in embryonic tissues; conversely, the maternal gDMR remains intact throughout the post-implantation epigenetic programming in extra-embryonic tissues (Kobayashi et al., 2013; Greenberg et al., 2017). Finally, the canonical $Z d b f 2$ promoter and exons remain silenced because of the incomplete establishment of the paternal sDMR, and the paternal expression of $\mathrm{Liz}$ continues throughout placental development (Greenberg et al., 2017). Thus far, it remains unclear why maternal gDMR persists in extra-embryonic tissues but not in embryos.

Paternal DNA methylation at the $Z d b f 2$ sDMR is required to prevent the accumulation of $\mathrm{H} 3 \mathrm{~K} 27 \mathrm{me} 3$, thereby conferring an active chromatin state at the adjacent $Z d b f 2$ promoter (Greenberg et al., 2017). It is not clearly understood what controls allele-specific DNA methylation at the sDMRs of canonical and non-canonical imprinted loci. However, further investigations into sDMRs at both canonical and non-canonical imprinted loci will provide valuable suggestions on how reprogramming or preserving factors target imprinted epigenetic marks through post-implantation development.

\section{CANONICAL AND NON-CANONICAL IMPRINTING IN THE OTHER RODENTS}

Although mice are the primary research model used to study genomic imprinting, imprinted regions have been described in various mammals, including humans. Among the 24 gDMRs in mice, two paternal (H19 and Dlk1-Meg3) and 16 maternal (Gprlas/Liz, Mcts2, Nnat, Nespas-Gnasxl, Gnas_exon1A, Peg10Sgce, Mest, Nap1l5, Peg3, Snrpn, Inpp5f_v2, Kcnq1ot1, Plagl1, Grb10, Peg13, and Airn) gDMRs were conserved between mice and humans (Table 1). Although some species-specific maternal gDMRs drive oocyte transcription initiation in lineage-specific LTR retrotransposons (Bogutz et al., 2019), many canonical imprinted loci are well conserved among species, and mice with deletions involving imprinted genes 
TABLE 1 | List of identified canonically and non-canonically imprinted regions.

\begin{tabular}{|c|c|c|c|c|}
\hline Type of imprinting & Mouse & Rat $^{\star 1}$ & Hamster*1 & Human \\
\hline Paternal gDMRs (canonical imprinting) & $\begin{array}{l}3 \text { loci (H19, } \\
\text { Dlk1-Meg3, } \\
\text { Rasgrf1) }\end{array}$ & $\begin{array}{l}3 \text { loci (H19, } \\
\text { Dlk1-Meg3, } \\
\text { Rasgrf1) }\end{array}$ & $\begin{array}{l}2 \text { loci }^{\star 2}(H 19, \\
\text { Dlk1-Meg3) }\end{array}$ & $\begin{array}{l}2 \text { loci (H19, } \\
\text { DLK1-MEG3) }\end{array}$ \\
\hline Maternal gDMRs (canonical imprinting) & $\begin{array}{l}16 \text { common } \\
\text { and } 5 \text { mouse } \\
\text { (rodent)- } \\
\text { specific loci } \\
\text { (Fkbp6, Cdh15, } \\
\text { Zrsr1, Slc38a4, } \\
\text { Impact) }\end{array}$ & $\begin{array}{l}\text { lgf2r, Peg3 } \\
\text { (common) and } \\
\text { Impact (rodent- } \\
\text { specific) }\end{array}$ & $\begin{array}{l}6 \text { common loci } \\
\text { (Peg3, etc.) and } \\
\text { Impact (rodent- } \\
\text { specific) }\end{array}$ & $\begin{array}{l}16 \text { common } \\
\text { and numerous } \\
\text { human-specific } \\
\text { loci }\end{array}$ \\
\hline Non-canonical imprinting & $\begin{array}{l}7 \text { loci (Sfmbt2, } \\
\text { Smoc1, Gab1, } \\
\text { etc.) }\end{array}$ & Sfmbt2 & Smoc1 & $\begin{array}{l}5 \text { loci } \\
\text { (FAM101A, } \\
\text { etc.) }\end{array}$ \\
\hline
\end{tabular}

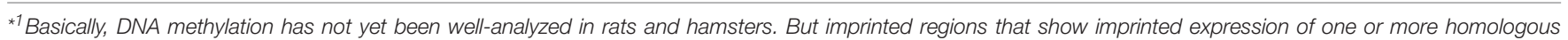
genes at each differentially methylated region (DMR) locus are listed.

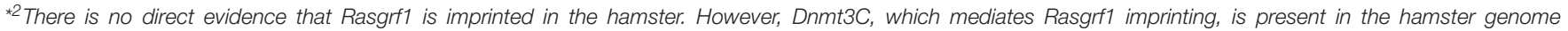
(Barau et al., 2016).

or ICRs are used as models for human imprinting diseases such as Prader-Willi, Angelman, Beckwith-Wiedemann, and Silver-Russell syndromes (Chang and Bartolomei, 2020). However, orthologs of non-canonical imprinted genes are not likely to be imprinted in humans. Preliminary studies in human embryos found five paternally expressed genes that may be regulated by maternal $\mathrm{H} 3 \mathrm{~K} 27 \mathrm{me} 3$, but none of these have been reported to be imprinted in mice (Zhang et al., 2019). Thus, current studies to date do not provide any direct evidence for the existence of non-canonical imprinting in mammals other than mice. However, among these genes, Sfmbt2 and Smoc1 have been reported to show an expression biased toward one parental allele in rat and hamster placentas, respectively (Wang et al., 2011; Brekke et al., 2016). This evidence supports the hypothesis that non-canonical imprinting is conserved in rodents.

Although rats and hamsters are widely used for physiological, oncological, and other medical studies, mice have always been used as embryological and genetic studies models. In this situation, the number of imprinted genes identified in these rodents is limited compared to mice. However, because of the long history of laboratory animal research, numerous mouse, rat, and hamster strains have been established and maintained, and the genomes of some have been sequenced. It is possible to identify imprinting information from polymorphism information among strains (Hermsen et al., 2015).

It has already been shown that single or multiple genes are imprinted on the homologous regions of the three imprinted clusters (H19, Dlk1-Meg3, and Rasgrf1) that undergo paternal methylation imprinting in mice (Overall et al., 1997; Pearsall et al., 1999; Dietz et al., 2012). In addition, Igf2r, Impact [driven by a rodent-specific LTR (Bogutz et al., 2019)], and $S f m b t 2$, which are controlled by maternal imprinting in mice, are also expressed only from one parental allele in rats (Mills et al., 1998; Okamura et al., 2005; Miri et al., 2013).
Sfmbt2 is almost exclusively expressed in extra-embryonic tissues and is essential for the maintenance of trophoblast progenitors. Intriguingly, Sfmbt2 contains a large cluster of microRNA (miRNA) genes within intron 10, and these miRNAs are also imprinted and essential for placental development (Inoue et al., 2017c). Notably, Sfmbt2, known to undergo non-canonical imprinting in mice, is also paternally expressed in the rat placenta in the presence of a large cluster of microRNAs (Wang et al., 2011). However, human, bovine, and pig SFMBT2 are not imprinted and lack this block of microRNAs. These observations strengthen the argument for the recent evolution of $S f m b t 2$, in which the non-canonical imprint (and the block of miRNAs) drives its placental role in rodents.

In hamsters, reciprocal crosses between two dwarf hamsters (Phodopus sungorus and Phodopus campbelli) result in strong parent-of-origin effects on placental and embryonic growth (Brekke and Good, 2014). The expression of imprinted genes and related loss-of-imprinting has been evaluated to some extent in dwarf hamster hybrids (Brekke et al., 2016). Singlenucleotide variant-based allele-specific analysis of placental expressed genes identified 88 imprinted candidate genes in hamster autosomes. Among these, 18 genes overlapped between hamster and mice, including Smoc1, a non-canonical imprinted gene. Unexpectedly, Smocl shows the opposite pattern of imprinting in hamster compared to mouse, with the maternal allele being expressed. This is similar to a report in human fibroblast cells, where SMOC1 showed maternal-allele specific expression (Santoni et al., 2017). While it is unclear whether this change is due to biological differences or false bias of allele-specific analysis, well-known examples of canonical paternally (Dlk1, Igf2, Impact, among others) and maternally (such as H19) expressed genes were also reidentified in hamster. Smoc1 encodes a multi-domain secreted protein that may play a critical role in ocular and limb development (Okada et al., 2011). However, Smoc1 is not likely associated with loss-of-imprinting in hybrid 
hamsters, and its functional role in placental development remains unknown.

\section{CONCLUSION}

The discovery of a non-canonical imprinting mechanism mediated by histone modifications is an important finding that provides a new molecular mechanism for epigenetic transgenerational inheritance. In contrast, the diversity of canonical and non-canonical imprinting complicates our understanding of the underlying mechanisms and a better understanding of the differences among mammalian species that bridge the gap between humans and mice. For instance, the insertion of endogenous retroviral elements drives both canonical and non-canonical imprinting (Bogutz et al., 2019; Hanna et al., 2019). However, not all species-specific imprinted regions can be explained by this mechanism. Revealing the whole landscape of genomic imprinting in various rodents, such as rats and hamsters, and non-human primates would be a significant

\section{REFERENCES}

Barau, J., Teissandier, A., Zamudio, N., Roy, S., Nalesso, V., Herault, Y., et al. (2016). The DNA methyltransferase DNMT3C protects male germ cells from transposon activity. Science 354, 909-912. doi: 10.1126/science.aah5143

Barlow, D. P., Stoger, R., Herrmann, B. G., Saito, K., and Schweifer, N. (1991). The mouse insulin-like growth factor type-2 receptor is imprinted and closely linked to the Tme locus. Nature 349, 84-87. doi: 10.1038/349084a0

Bartolomei, M. S., Zemel, S., and Tilghman, S. M. (1991). Parental imprinting of the mouse H19 gene. Nature 351, 153-155. doi: 10.1038/351153a0

Barton, S. C., Surani, M. A., and Norris, M. L. (1984). Role of paternal and maternal genomes in mouse development. Nature 311, 374-376. doi: 10.1038/311374a0

Baubec, T., Colombo, D. F., Wirbelauer, C., Schmidt, J., Burger, L., Krebs, A. R., et al. (2015). Genomic profiling of DNA methyltransferases reveals a role for DNMT3B in genic methylation. Nature 520, 243-247. doi: 10.1038/ nature 14176

Bogutz, A. B., Brind'Amour, J., Kobayashi, H., Jensen, K. N., Nakabayashi, K., Imai, H., et al. (2019). Evolution of imprinting via lineage-specific insertion of retroviral promoters. Nat. Commun. 10:5674. doi: 10.1038/s41467-019-13 $662-9$

Bourc'his, D., Xu, G. L., Lin, C. S., Bollman, B., and Bestor, T. H. (2001). Dnmt3L and the establishment of maternal genomic imprints. Science 294, 2536-2539. doi: $10.1126 /$ science. 1065848

Brekke, T. D., and Good, J. M. (2014). Parent-of-origin growth effects and the evolution of hybrid inviability in dwarf hamsters. Evolution 68, 3134-3148. doi: $10.1111 /$ evo. 12500

Brekke, T. D., Henry, L. A., and Good, J. M. (2016). Genomic imprinting, disrupted placental expression, and speciation. Evolution 70, 2690-2703. doi: 10.1111/evo. 13085

Brind'Amour, J., Kobayashi, H., Richard Albert, J., Shirane, K., Sakashita, A., Kamio, A., et al. (2018). LTR retrotransposons transcribed in oocytes drive species-specific and heritable changes in DNA methylation. Nat. Commun. 9, 3331. doi: 10.1038/s41467-018-05841-x

Cattanach, B. M. (1986). Parental origin effects in mice. J. Embryol. Exp. Morphol. 97, 137-150.

Chaillet, J. R., Vogt, T. F., Beier, D. R., and Leder, P. (1991). Parental-specific methylation of an imprinted transgene is established during gametogenesis and progressively changes during embryogenesis. Cell 66, 77-83. doi: 10.1016/00928674(91)90140-t

Chang, S., and Bartolomei, M. S. (2020). Modeling human epigenetic disorders in mice: Beckwith-Wiedemann syndrome and Silver-Russell syndrome. Dis. Model. Mech. 13:dmm044123. doi: 10.1242/dmm.044123 step forward in understanding the diversity of imprinting and epigenetic regulation systems.

\section{AUTHOR CONTRIBUTIONS}

The author confirms being the sole contributor of this work and has approved it for publication.

\section{FUNDING}

This research was supported by the JSPS KAKENHI (Grant Number JP21H02382 to HK).

\section{ACKNOWLEDGMENTS}

I would like to thank Editage (http://www.editage.jp) for English language editing.

Chen, Z., and Zhang, Y. (2020). Maternal H3K27me3-dependent autosomal and X chromosome imprinting. Nat. Rev. Genet. 21, 555-571. doi: 10.1038/s41576020-0245-9

Chen, Z., Djekidel, M. N., and Zhang, Y. (2021). Distinct dynamics and functions of H2AK119ub1 and H3K27me3 in mouse preimplantation embryos. Nat. Genet. 53, 551-563. doi: 10.1038/s41588-021-00821-2

Chen, Z., Yin, Q., Inoue, A., Zhang, C., and Zhang, Y. (2019). Allelic H3K27me3 to allelic DNA methylation switch maintains noncanonical imprinting in extraembryonic cells. Sci. Adv. 5:eaay7246. doi: 10.1126/sciadv.aay7246

Chotalia, M., Smallwood, S. A., Ruf, N., Dawson, C., Lucifero, D., Frontera, M., et al. (2009). Transcription is required for establishment of germline methylation marks at imprinted genes. Genes Dev. 23, 105-117. doi: 10.1101/ gad.495809

Davis, T. L., Yang, G. J., Mccarrey, J. R., and Bartolomei, M. S. (2000). The H19 methylation imprint is erased and re-established differentially on the parental alleles during male germ cell development. Hum. Mol. Genet. 9, 2885-2894. doi: 10.1093/hmg/9.19. 2885

DeChiara, T. M., Robertson, E. J., and Efstratiadis, A. (1991). Parental imprinting of the mouse insulin-like growth factor II gene. Cell 64, 849-859. doi: 10.1016/ 0092-8674(91)90513-x

Dietz, W. H., Masterson, K., Sittig, L. J., Redei, E. E., and Herzing, L. B. (2012). Imprinting and expression of Dio3os mirrors Dio3 in rat. Front. Genet. 3:279. doi: 10.3389/fgene.2012.00279

Duffie, R., Ajjan, S., Greenberg, M. V., Zamudio, N., Escamilla Del Arenal, M., Iranzo, J., et al. (2014). The Gpr1/Zdbf2 locus provides new paradigms for transient and dynamic genomic imprinting in mammals. Genes Dev. 28, 463478. doi: 10.1101/gad.232058.113

Fan, T., Hagan, J. P., Kozlov, S. V., Stewart, C. L., and Muegge, K. (2005). Lsh controls silencing of the imprinted Cdkn1c gene. Development 132, 635-644. doi: $10.1242 /$ dev.01612

Ferguson-Smith, A. C. (2011). Genomic imprinting: the emergence of an epigenetic paradigm. Nat. Rev. Genet. 12, 565-575. doi: 10.1038/nrg3032

Gibbs, R. A., Weinstock, G. M., Metzker, M. L., Muzny, D. M., Sodergren, E. J., Scherer, S., et al. (2004). Genome sequence of the Brown Norway rat yields insights into mammalian evolution. Nature 428, 493-521. doi: 10.1038/ nature 02426

Greenberg, M. V., Glaser, J., Borsos, M., Marjou, F. E., Walter, M., Teissandier, A., et al. (2017). Transient transcription in the early embryo sets an epigenetic state that programs postnatal growth. Nat. Genet. 49, 110-118. doi: 10.1038/ng.3718

Hanna, C. W., Perez-Palacios, R., Gahurova, L., Schubert, M., Krueger, F., Biggins, L., et al. (2019). Endogenous retroviral insertions drive non-canonical 
imprinting in extra-embryonic tissues. Genome Biol. 20:225. doi: 10.1186/ s13059-019-1833-x

Hark, A. T., Schoenherr, C. J., Katz, D. J., Ingram, R. S., Levorse, J. M., and Tilghman, S. M. (2000). CTCF mediates methylation-sensitive enhancerblocking activity at the H19/Igf2 locus. Nature 405, 486-489. doi: 10.1038/ 35013106

Hata, K., Okano, M., Lei, H., and Li, E. (2002). Dnmt3L cooperates with the Dnmt3 family of de novo DNA methyltransferases to establish maternal imprints in mice. Development 129, 1983-1993. doi: 10.1242/dev.129.8.1983

Hermsen, R., De Ligt, J., Spee, W., Blokzijl, F., Schafer, S., Adami, E., et al. (2015). Genomic landscape of rat strain and substrain variation. BMC Genom. 16:357. doi: 10.1186/s12864-015-1594-1

Hirasawa, R., Chiba, H., Kaneda, M., Tajima, S., Li, E., Jaenisch, R., et al. (2008). Maternal and zygotic Dnmtl are necessary and sufficient for the maintenance of DNA methylation imprints during preimplantation development. Genes Dev. 22, 1607-1616. doi: 10.1101/gad.1667008

Hiura, H., Obata, Y., Komiyama, J., Shirai, M., and Kono, T. (2006). Oocyte growth-dependent progression of maternal imprinting in mice. Genes Cells 11, 353-361. doi: 10.1111/j.1365-2443.2006.00943.x

Inoue, A., Jiang, L., Lu, F., Suzuki, T., and Zhang, Y. (2017a). Maternal H3K27me3 controls DNA methylation-independent imprinting. Nature 547, 419-424. doi: 10.1038 /nature23262

Inoue, A., Jiang, L., Lu, F., and Zhang, Y. (2017b). Genomic imprinting of Xist by maternal H3K27me3. Genes Dev. 31, 1927-1932. doi: 10.1101/gad.304113.117

Inoue, K., Hirose, M., Inoue, H., Hatanaka, Y., Honda, A., Hasegawa, A., et al. (2017c). The Rodent-Specific MicroRNA Cluster within the Sfmbt2 Gene Is Imprinted and Essential for Placental Development. Cell. Rep. 19, 949-956. doi: 10.1016/j.celrep.2017.04.018

Itoh, M., Yoshida, Y., Nishida, K., Narimatsu, M., Hibi, M., and Hirano, T. (2000). Role of Gabl in heart, placenta, and skin development and growth factorand cytokine-induced extracellular signal-regulated kinase mitogen-activated protein kinase activation. Mol. Cell. Biol. 20, 3695-3704. doi: 10.1128/mcb.20. 10.3695-3704.2000

John, R. M., and Lefebvre, L. (2011). Developmental regulation of somatic imprints. Differentiation 81, 270-280. doi: 10.1016/j.diff.2011.01.007

Johnson, D. R. (1974). Hairpin-tail: a case of post-reductional gene action in the mouse egg. Genetics 76, 795-805.

Kajii, T., and Ohama, K. (1977). Androgenetic origin of hydatidiform mole. Nature 268, 633-634. doi: 10.1038/268633a0

Kalish, J. M., Jiang, C., and Bartolomei, M. S. (2014). Epigenetics and imprinting in human disease. Int. J. Dev. Biol. 58, 291-298. doi: 10.1387/ijdb.140077mb

Kaneda, M., Okano, M., Hata, K., Sado, T., Tsujimoto, N., Li, E., et al. (2004). Essential role for de novo DNA methyltransferase Dnmt3a in paternal and maternal imprinting. Nature 429, 900-903. doi: 10.1038/nature02633

Kato, Y., Kaneda, M., Hata, K., Kumaki, K., Hisano, M., Kohara, Y., et al. (2007). Role of the Dnmt3 family in de novo methylation of imprinted and repetitive sequences during male germ cell development in the mouse. Hum. Mol. Genet. 16, 2272-2280. doi: 10.1093/hmg/ddm 179

Kawahara, M., Wu, Q., Takahashi, N., Morita, S., Yamada, K., Ito, M., et al. (2007). High-frequency generation of viable mice from engineered bi-maternal embryos. Nat. Biotechnol. 25, 1045-1050. doi: 10.1038/nbt1331

Kobayashi, H., Sakurai, T., Imai, M., Takahashi, N., Fukuda, A., Yayoi, O., et al. (2012a). Contribution of intragenic DNA methylation in mouse gametic DNA methylomes to establish oocyte-specific heritable marks. PLoS Genet. 8:e1002440. doi: 10.1371/journal.pgen.100 2440

Kobayashi, H., Sakurai, T., Sato, S., Nakabayashi, K., Hata, K., and Kono, T. (2012b). Imprinted DNA methylation reprogramming during early mouse embryogenesis at the Gpr1-Zdbf2 locus is linked to long cis-intergenic transcription. FEBS Lett. 586, 827-833. doi: 10.1016/j.febslet.2012.01.059

Kobayashi, H., Yamada, K., Morita, S., Hiura, H., Fukuda, A., Kagami, M., et al. (2009). Identification of the mouse paternally expressed imprinted gene Zdbf2 on chromosome 1 and its imprinted human homolog ZDBF2 on chromosome 2. Genomics 93, 461-472. doi: 10.1016/j.ygeno.2008.12.012

Kobayashi, H., Yanagisawa, E., Sakashita, A., Sugawara, N., Kumakura, S., Ogawa, H., et al. (2013). Epigenetic and transcriptional features of the novel human imprinted IncRNA GPR1AS suggest it is a functional ortholog to mouse Zdbf2linc. Epigenetics 8, 635-645. doi: 10.4161/epi.24887
Kono, T., Obata, Y., Wu, Q., Niwa, K., Ono, Y., Yamamoto, Y., et al. (2004). Birth of parthenogenetic mice that can develop to adulthood. Nature 428, 860-864. doi: 10.1038/nature02402

Latos, P. A., Pauler, F. M., Koerner, M. V., Senergin, H. B., Hudson, Q. J., Stocsits, R. R., et al. (2012). Airn transcriptional overlap, but not its IncRNA products, induces imprinted Igf2r silencing. Science 338, 1469-1472. doi: 10.1126/science. 1228110

Lewis, A., Mitsuya, K., Umlauf, D., Smith, P., Dean, W., Walter, J., et al. (2004). Imprinting on distal chromosome 7 in the placenta involves repressive histone methylation independent of DNA methylation. Nat. Genet. 36, 1291-1295. doi: $10.1038 / \mathrm{ng} 1468$

Li, Z. K., Wang, L. Y., Wang, L. B., Feng, G. H., Yuan, X. W., Liu, C., et al. (2018). Generation of Bimaternal and Bipaternal Mice from Hypomethylated Haploid ESCs with Imprinting Region Deletions. Cell Stem Cell 23:e664. doi: 10.1016/j.stem.2018.09.004

Linder, D., Mccaw, B. K., and Hecht, F. (1975). Parthenogenic origin of benign ovarian teratomas. N. Engl. J. Med. 292, 63-66. doi: 10.1056/ NEJM197501092920202

Lopes, S., Lewis, A., Hajkova, P., Dean, W., Oswald, J., Forne, T., et al. (2003). Epigenetic modifications in an imprinting cluster are controlled by a hierarchy of DMRs suggesting long-range chromatin interactions. Hum. Mol. Genet. 12, 295-305. doi: 10.1093/hmg/ddg022

Lucifero, D., Mann, M. R., Bartolomei, M. S., and Trasler, J. M. (2004). Genespecific timing and epigenetic memory in oocyte imprinting. Hum. Mol. Genet. 13, 839-849. doi: 10.1093/hmg/ddh104

Matoba, S., Nakamuta, S., Miura, K., Hirose, M., Shiura, H., Kohda, T., et al. (2019). Paternal knockout of Slc38a4/SNAT4 causes placental hypoplasia associated with intrauterine growth restriction in mice. Proc. Natl. Acad. Sci. U. S. A. 116, 21047-21053. doi: 10.1073/pnas.1907884116

McGrath, J., and Solter, D. (1984). Completion of mouse embryogenesis requires both the maternal and paternal genomes. Cell 37, 179-183. doi: 10.1016/00928674(84)90313-1

Mei, H., Kozuka, C., Hayashi, R., Kumon, M., Koseki, H., and Inoue, A. (2021). H2AK119ub1 guides maternal inheritance and zygotic deposition of H3K27me3 in mouse embryos. Nat. Genet. 53, 539-550. doi: 10.1038/s41588021-00820-3

Messerschmidt, D. M., De Vries, W., Ito, M., Solter, D., Ferguson-Smith, A., and Knowles, B. B. (2012). Trim 28 is required for epigenetic stability during mouse oocyte to embryo transition. Science 335, 1499-1502. doi: 10.1126/science. 1216154

Mills, J. J., Falls, J. G., De Souza, A. T., and Jirtle, R. L. (1998). Imprinted M6p/Igf2 receptor is mutated in rat liver tumors. Oncogene 16, 2797-2802. doi: 10.1038/ sj.onc. 1201801

Miri, K., Latham, K., Panning, B., Zhong, Z., Andersen, A., and Varmuza, S. (2013). The imprinted polycomb group gene Sfmbt2 is required for trophoblast maintenance and placenta development. Development 140, 4480-4489. doi: 10.1242/dev.096511

Mohammad, F., Pandey, G. K., Mondal, T., Enroth, S., Redrup, L., Gyllensten, U., et al. (2012). Long noncoding RNA-mediated maintenance of DNA methylation and transcriptional gene silencing. Development 139, 2792-2803. doi: 10.1242/ dev.079566

Moore, T., and Haig, D. (1991). Genomic imprinting in mammalian development: a parental tug-of-war. Trends Genet. 7, 45-49. doi: 10.1016/0168-9525(91) 90230-N

Okada, I., Hamanoue, H., Terada, K., Tohma, T., Megarbane, A., Chouery, E., et al. (2011). SMOC1 is essential for ocular and limb development in humans and mice. Am. J. Hum. Genet. 88, 30-41. doi: 10.1016/j.ajhg.2010.11.012

Okae, H., Matoba, S., Nagashima, T., Mizutani, E., Inoue, K., Ogonuki, N., et al. (2014). RNA sequencing-based identification of aberrant imprinting in cloned mice. Hum. Mol. Genet. 23, 992-1001. doi: 10.1093/hmg/ddt495

Okamura, K., Sakaki, Y., and Ito, T. (2005). Comparative genomics approach toward critical determinants for the imprinting of an evolutionarily conserved gene Impact. Biochem. Biophys. Res. Commun. 329, 824-830. doi: 10.1016/j. bbrc.2005.02.048

Overall, M., Bakker, M., Spencer, J., Parker, N., Smith, P., and Dziadek, M. (1997). Genomic imprinting in the rat: linkage of Igf2 and H19 genes and opposite parental allele-specific expression during embryogenesis. Genomics 45, 416-420. doi: 10.1006/geno.1997.4933 
Pearsall, R. S., Plass, C., Romano, M. A., Garrick, M. D., Shibata, H., Hayashizaki, Y., et al. (1999). A direct repeat sequence at the Rasgrf1 locus and imprinted expression. Genomics 55, 194-201. doi: 10.1006/geno.1998.5660

Quenneville, S., Verde, G., Corsinotti, A., Kapopoulou, A., Jakobsson, J., Offner, S., et al. (2011). In embryonic stem cells, ZFP57/KAP1 recognize a methylated hexanucleotide to affect chromatin and DNA methylation of imprinting control regions. Mol. Cell .44, 361-372. doi: 10.1016/j.molcel.2011.08.032

Saito, T., Hara, S., Kato, T., Tamano, M., Muramatsu, A., Asahara, H., et al. (2018). A tandem repeat array in IG-DMR is essential for imprinting of paternal allele at the Dlk1-Dio3 domain during embryonic development. Hum. Mol. Genet. 27, 3283-3292. doi: 10.1093/hmg/ddy235

Santoni, F. A., Stamoulis, G., Garieri, M., Falconnet, E., Ribaux, P., Borel, C., et al. (2017). Detection of Imprinted Genes by Single-Cell Allele-Specific Gene Expression. Am. J. Hum. Genet. 100, 444-453. doi: 10.1016/j.ajhg.2017.01.028

Sato, S., Yoshida, W., Soejima, H., Nakabayashi, K., and Hata, K. (2011). Methylation dynamics of IG-DMR and Gtl2-DMR during murine embryonic and placental development. Genomics 98, 120-127. doi: 10.1016/j.ygeno.2011. 05.003

Sharif, J., Muto, M., Takebayashi, S., Suetake, I., Iwamatsu, A., Endo, T. A., et al. (2007). The SRA protein Np95 mediates epigenetic inheritance by recruiting Dnmt1 to methylated DNA. Nature 450, 908-912. doi: 10.1038/nature06397

Shirane, K., Miura, F., Ito, T., and Lorincz, M. C. (2020). NSD1-deposited H3K36me2 directs de novo methylation in the mouse male germline and counteracts Polycomb-associated silencing. Nat. Genet. 52, 1088-1098. doi: 10.1038/s41588-020-0689-z

Smallwood, S. A., Tomizawa, S., Krueger, F., Ruf, N., Carli, N., Segonds-Pichon, A., et al. (2011). Dynamic CpG island methylation landscape in oocytes and preimplantation embryos. Nat. Genet. 43, 811-814. doi: 10.1038/ng.864

Stoger, R., Kubicka, P., Liu, C. G., Kafri, T., Razin, A., Cedar, H., et al. (1993). Maternal-specific methylation of the imprinted mouse Igf $2 \mathrm{r}$ locus identifies the expressed locus as carrying the imprinting signal. Cell 73, 61-71. doi: 10.1016/ 0092-8674(93)90160-r

Takahashi, N., Coluccio, A., Thorball, C. W., Planet, E., Shi, H., Offner, S., et al. (2019). ZNF445 is a primary regulator of genomic imprinting. Genes Dev. 33, 49-54. doi: 10.1101/gad.320069.118

Terranova, R., Yokobayashi, S., Stadler, M. B., Otte, A. P., Van Lohuizen, M., Orkin, S. H., et al. (2008). Polycomb group proteins Ezh2 and Rnf2 direct genomic contraction and imprinted repression in early mouse embryos. Dev. Cell. 15, 668-679. doi: 10.1016/j.devcel.2008.08.015

Trivers, R. L. (1974). Parent-Offspring Conflict. Am. Zool. 14, 249-264. doi: 10. 1093/icb/14.1.249

Tucci, V., Isles, A. R., Kelsey, G., Ferguson-Smith, A. C., and Erice Imprinting, G. (2019). Genomic Imprinting and Physiological Processes in Mammals. Cell 176, 952-965. doi: 10.1016/j.cell.2019.01.043

Veselovska, L., Smallwood, S. A., Saadeh, H., Stewart, K. R., Krueger, F., MaupetitMehouas, S., et al. (2015). Deep sequencing and de novo assembly of the mouse oocyte transcriptome define the contribution of transcription to the DNA methylation landscape. Genome Biol. 16:209. doi: 10.1186/s13059-015-0769-z

Wake, N., Takagi, N., and Sasaki, M. (1978). Androgenesis as a cause of hydatidiform mole. J. Natl. Cancer Inst. 60, 51-57. doi: 10.1093/jnci/60.1.51
Wang, Q., Chow, J., Hong, J., Smith, A. F., Moreno, C., Seaby, P., et al. (2011). Recent acquisition of imprinting at the rodent Sfmbt2 locus correlates with insertion of a large block of miRNAs. BMC Genom. 12:204. doi: 10.1186/14712164-12-204

Watanabe, T., Tomizawa, S., Mitsuya, K., Totoki, Y., Yamamoto, Y., Kuramochi-Miyagawa, S., et al. (2011). Role for piRNAs and noncoding RNA in de novo DNA methylation of the imprinted mouse Rasgrf1 locus. Science 332, 848-852. doi: 10.1126/science.120 3919

Waterston, R. H., Lindblad-Toh, K., Birney, E., Rogers, J., Abril, J. F., Agarwal, P., et al. (2002). Initial sequencing and comparative analysis of the mouse genome. Nature 420, 520-562. doi: 10.1038/nature01262

Williamson, C. M., Ball, S. T., Dawson, C., Mehta, S., Beechey, C. V., Fray, M., et al. (2011). Uncoupling antisense-mediated silencing and DNA methylation in the imprinted Gnas cluster. PLoS Genet. 7:e1001347. doi: 10.1371/journal. pgen.1001347

Winking, H., and Silver, L. M. (1984). Characterization of a recombinant mouse T haplotype that expresses a dominant lethal maternal effect. Genetics 108, 1013-1020.

Wood, M. D., Hiura, H., Tunster, S. J., Arima, T., Shin, J. Y., Higgins, M. J., et al. (2010). Autonomous silencing of the imprinted Cdkn1c gene in stem cells. Epigenetics 5, 214-221. doi: 10.4161/epi.5.3.1 1275

Xu, Q., Xiang, Y., Wang, Q., Wang, L., Brind'amour, J., Bogutz, A. B., et al. (2019). SETD2 regulates the maternal epigenome, genomic imprinting and embryonic development. Nat. Genet. 51, 844-856. doi: 10.1038/s41588-019-0398-7

Yamasaki, Y., Kayashima, T., Soejima, H., Kinoshita, A., Yoshiura, K., Matsumoto, N., et al. (2005). Neuron-specific relaxation of Igf2r imprinting is associated with neuron-specific histone modifications and lack of its antisense transcript Air. Hum. Mol. Genet. 14, 2511-2520. doi: 10.1093/hmg/ddi255

Zhang, W., Chen, Z., Yin, Q., Zhang, D., Racowsky, C., and Zhang, Y. (2019). Maternal-biased H3K27me3 correlates with paternal-specific gene expression in the human morula. Genes Dev. 33, 382-387. doi: 10.1101/gad.323105.118

Conflict of Interest: The author declares that the research was conducted in the absence of any commercial or financial relationships that could be construed as a potential conflict of interest.

Publisher's Note: All claims expressed in this article are solely those of the authors and do not necessarily represent those of their affiliated organizations, or those of the publisher, the editors and the reviewers. Any product that may be evaluated in this article, or claim that may be made by its manufacturer, is not guaranteed or endorsed by the publisher.

Copyright (c) 2021 Kobayashi. This is an open-access article distributed under the terms of the Creative Commons Attribution License (CC BY). The use, distribution or reproduction in other forums is permitted, provided the original author(s) and the copyright owner(s) are credited and that the original publication in this journal is cited, in accordance with accepted academic practice. No use, distribution or reproduction is permitted which does not comply with these terms. 